\title{
MULTI-MODAL IMPEDANCE SPECTROSCOPY DEVICE FOR SIMULTANEOUS MEASUREMENT OF ELECTRICAL AND MECHANICAL PROPERTIES OF CELLS
}

\author{
Karthik Mahesh ${ }^{1}$, Kshama Parate ${ }^{2}$, Charan M. Shah ${ }^{3}$, Manoj M. Varmal and Prosenjit Sen ${ }^{1}$ \\ ${ }^{1}$ Centre for Nano Science and Engineering (CeNSE), Indian Institute of Science, Bangalore, India \\ ${ }^{2}$ Department of Mechanical Engineering, Iowa State University, Iowa, United States of America \\ ${ }^{3}$ Functional Materials and Microsystems Group, Royal Melbourne Institute of Technology, Australia
}

\begin{abstract}
We demonstrate a novel microfluidic device based on electrical impedance spectroscopy (EIS) which independently measures the electrical properties of cells and simultaneously estimates their mechanical properties by measuring transit times across a mechanical constriction. The device also allows electrically probing the effect of mechanical squeezing on the cell. Compared to conventional EIS techniques that measures the electrical impedance of cells solely across a constriction, the proposed technique offers more versatility and enables multi-modal analysis of cellular properties.
\end{abstract}

\section{INTRODUCTION}

Electrical properties of single cells are conventionally studied using microfluidic coulter counters, which monitor the impedance of a small orifice as the cells pass through it. Due to its simple architecture, limited use of different signal frequencies and absence of self-referenced measurement, this technique was primarily restricted to counting and sizing of different cell types. In contrast, microfluidic electrical impedance spectroscopy (EIS) is a label-free non-invasive method to estimate the biological state of a cell by measuring its dielectric properties at multiple frequencies simultaneously. The technique involves measuring the electrical impedance of the cells flowing through a pair of microfabricated electrodes within a microchannel. The excitation signal is usually an AC voltage, $\boldsymbol{V}(j \omega)$. The current response, $\boldsymbol{I}(j \omega)$, is measured and the impedance, $\boldsymbol{Z}(j \omega)$, of the system is given as:

$$
\frac{\boldsymbol{V}(j \omega)}{\boldsymbol{I}(j \omega)}=\boldsymbol{Z}(j \omega)=\boldsymbol{Z}_{\text {real }}+\boldsymbol{Z}_{\text {img }}
$$

where $\boldsymbol{Z}_{\text {real }}$ and $\boldsymbol{Z}_{\text {img }}$ are the real and imaginary parts of the impedance at the excitation frequency $\omega$ [2]. In order to obtain a detailed view of the cells' biological state, the electrical impedance is often measured simultaneously at multiple frequencies. For the most commonly used electrode designs, the lower limit of the frequency is limited to $\sim 100 \mathrm{kHz}$ due to the electrical double layer capacitance at the electrode interface. The higher frequency is limited by the readout electronics with frequencies up to $90 \mathrm{MHz}$ being used to distinguish fungal cells [1]. Recent advances in this technique have enabled high throughput analysis (1000 cells per second) of a large population of cells at a single cell level $[2,3]$.
Recently deformability cytometry has been developed for high-throughput measurement of the mechanical properties of cells at a single cell level $[4,5]$. In this technique, cells flowing through a microchannel are mechanically stretched either using the flow or using a mechanical constriction. By measuring the deformation of the cell and the time that the cell requires to traverse the constriction (i.e. transit time) it is possible to qualitatively assess the cells' mechanical properties. For measuring the deformation of the cell, these works relied on the high speed imaging technique, requiring an expensive setup.

Using the coulter counter configuration, previous studies [6-8] have classified cell types by measuring the cells impedance in a constricted channel. By monitoring the time variation of impedance, the transit time was measured to estimate the cells mechanical property. These studies were however limited, as they were able to measure cell impedance only in the squeezed state and hence provided coupled information only. We report a truly multimodal measurement technique which independently measures the electrical properties of undeformed cells and estimates their mechanical properties by measuring transit times through a constriction. The platform also allows measurement of the change in properties of cells due to the mechanical squeezing when it passes through a constriction. This makes our system uniquely versatile and has not been reported elsewhere.

Cell stiffness is often associated with diseases such as diabetes, hypertension, myocardial infarction and other cardiovascular diseases [9, 10]. In specific cases, cell stiffness has been shown to change with its developmental stage. Hence, measurement of cells mechanical properties becomes a unique method to characterize distribution of development stages in a population of cells [4]. It is well reported in literature that glutaraldehyde, a chemical fixative, contributes to increasing cell stiffness by acting on all the structural components of the cell: the lipid membrane, the fluid cytoplasm, and the membrane skeleton. Glutaraldehyde also increases the effective viscosity of the cytoplasm and lipid membrane. Using chemically stiffened cells, we demonstrate differences in signal magnitudes and transit times between healthy and glutaraldehyde treated cells [11].

\section{DEVICE CONCEPT}

In order to measure cell impedance in a selfreferenced manner [1, 2], two sets of Au electrodes are used. The electrodes are designed in such a way that one of the electrodes in each set acts as the source and the other one acts as the reference electrode. With the 
assumption that only one cell passes over the electrode set at any given time, a self-reference measurement is more immune to measurement drifts and changes in medium conductivity. In our design, the gap between one electrode pair to another was $200 \mu \mathrm{m}$ and between adjacent electrodes was $20 \mu \mathrm{m}$ as seen in Figure 1.

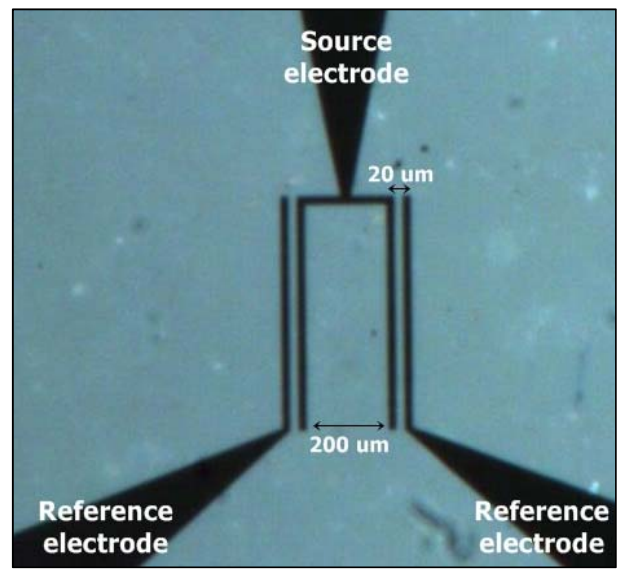

Figure 1: Electrode configuration

Figure 2 shows the concept of our technique. Similar to a conventional EIS setup $[1,6,7]$, the impedance of the cells passing over the electrodes were measured in a selfreferenced manner. This technique leads to two peaks (positive/negative) in the measurement signal, corresponding to the electrode over which the cell is passing. As shown in Figure 2 the main innovation in this report is to place the mechanical constriction in between the electrode pairs. In this design by measuring the time difference between the positive and the negative peaks (i.e. transit time) we were able to estimate the mechanical properties of the squeezed cells. The values of the individual peaks (positive/negative) corresponds to the dielectric properties of the cell before and after the constriction and hence allows investigation of the effect of mechanical squeezing on the cell.

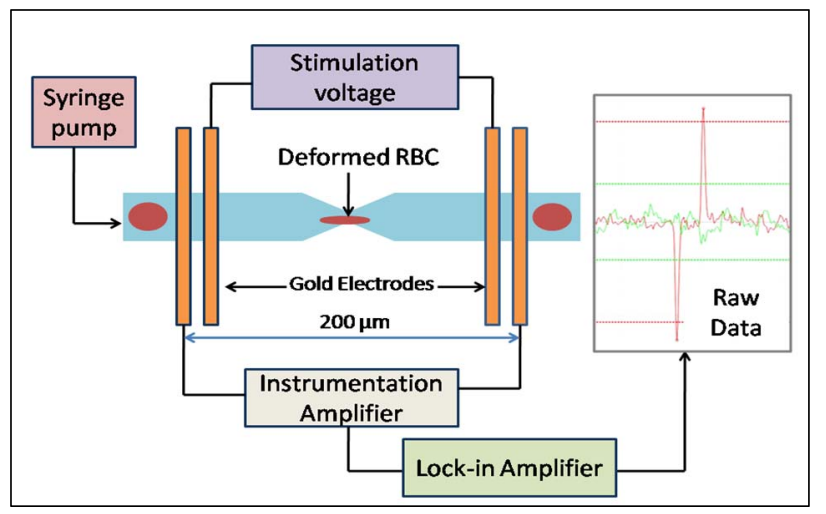

Figure 2: Schematic of the device design and the experimental technique

\section{DEVICE FABRICATION}

Glass substrates were cleaned in Piranha solution (3:1 $\mathrm{H}_{2} \mathrm{SO}_{4}: \mathrm{H}_{2} \mathrm{O}_{2}$ ) for ten minutes to remove organic and metallic contaminants. They were rinsed with DI water and dried with $\mathrm{N}_{2}$ gas. After a one minute dehydration bake at $250^{\circ} \mathrm{C}, 20 / 100 \mathrm{~nm}$ of $\mathrm{Cr} / \mathrm{Au}$ was deposited on the glass substrates using Tecport $^{\circledR}$ sputtering tool. Next, a positive photoresist S1813 was spin coated at 3000 rpm for $30 \mathrm{~s}$. Following a soft bake at $95^{\circ} \mathrm{C}$, photolithography was done at $45 \mathrm{~mJ} / \mathrm{cm}^{2}$ using a mask aligner (EVG-620). The slides were developed in MF26A and hard baked at $110^{\circ} \mathrm{C}$. Wet etching was done by immersing the slides in $\mathrm{Au}$ etchant $\left(4: 1: 40 \mathrm{KI}: \mathrm{I}_{2}: \mathrm{DI}\right.$ water) for $30 \mathrm{~s}$ and $\mathrm{Cr}$ etchant for $10 \mathrm{~s}$. The electrode fabrication procedure was completed by rinsing the substrates in acetone to remove the photoresist, followed by a DI water rinse, and blow drying them with $\mathrm{N}_{2}$ gas.

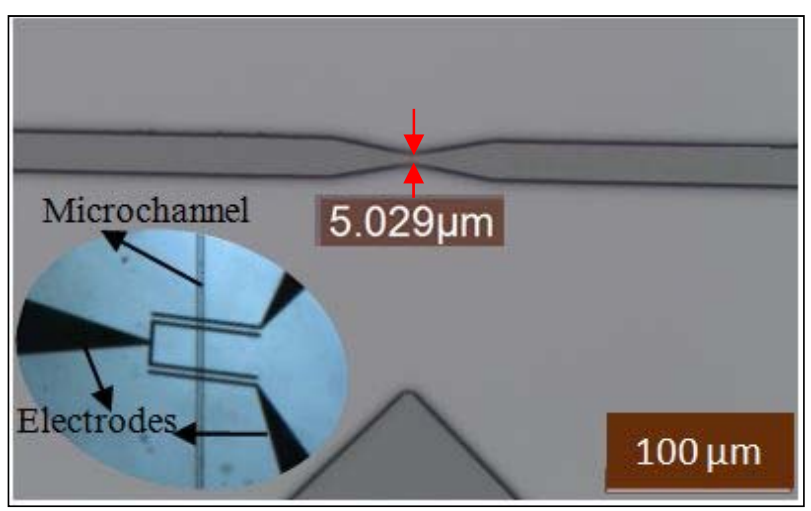

Figure 3: $5 \mu \mathrm{m}$ constriction in the microchannel, post SU8 lithography; Inset: Gold electrodes integrated with the PDMS microchannel

A master mold for the microfluidic channels was fabricated using standard SU-8 2015 lithography. The channels were designed to have a depth of $10 \mu \mathrm{m}$, a width of $20 \mu \mathrm{m}$ and length of $1.5 \mathrm{~cm}$. A $5 \mu \mathrm{m}$ mechanical constriction was designed to mechanically squeeze the cells (see Figure 3). Using the SU-8 molds, PDMS (polydimethylsiloxane) microchannels were fabricated. For bonding, both the PDMS devices and the electrodes were plasma treated for a minute. The microchannels were aligned with the electrodes under a microscope and irreversibly bonded to form one complete unit (Figure 3 Inset). Standard tubing was inserted into the inlet and outlet ports to introduce the sample.

\section{EXPERIMENTAL SETUP}

A $800 \mathrm{kHz}$ signal was used to stimulate the electrodes in the channel. Signal captured from the reference electrodes were subtracted using a custom-built instrumentation amplifier and measured using a lock-in amplifier (HF2LI, Zurich Instruments). Analog Devices operational amplifier (AD8066) was used to design and build the low noise instrumentation amplifier. As seen in Figure 4 the board was designed such that the microfluidic chip could be mounted on the board to reduce parasitic effects.

Red blood cells (RBCs) were used in our experiments. We used both healthy and artificially stiffened red blood cells RBCs (glutaraldehyde treated). The cells were diluted with PBS, loaded into a syringe, and driven through the channel using a syringe pump at a flow rate of $50 \mu \mathrm{l} / \mathrm{min}$. Raw data captured using the lock- 
in amplifier was processed using a custom code to automatically identify the cell transit events. For each transit, we measured and recorded the peak values and the time difference between the peaks (i.e transit time).

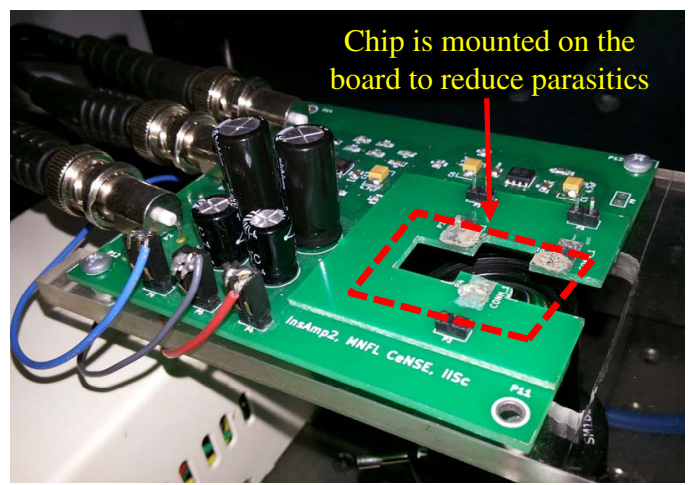

Figure 4: Image of the custom-built instrumentation amplifier

\section{EXPERIMENTAL RESULTS}
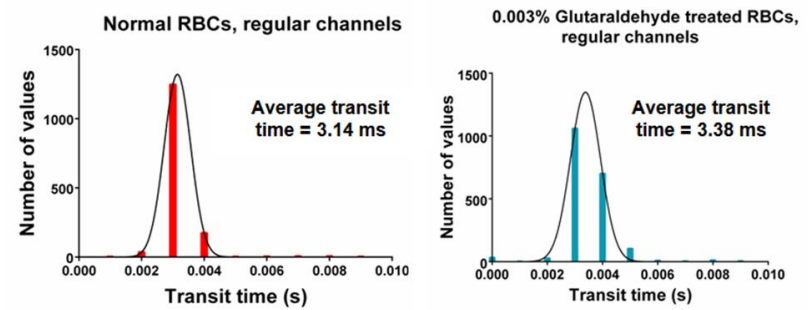

Figure 5: Transit times of normal RBCs and glutaraldehyde treated $R B C s$ in regular channels

The experimental system was verified by passing normal and glutaraldehyde treated RBCs in regular microchannels without any constriction. As seen in Figure 5 , the transit times are almost similar. The measured transit time matches well with the theoretical value expected for the used flow rates.
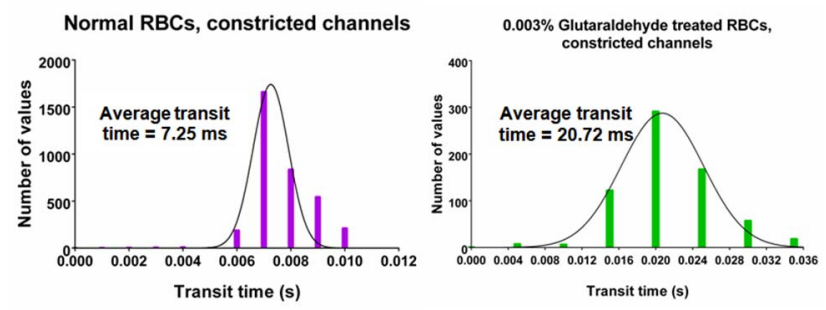

Figure 6: Transit times of normal RBCs and glutaraldehyde treated RBCs in constricted channels

To obtain information on the mechanical properties of the cell, we measured the transit time for both normal RBCs and glutaraldehyde treated RBCs in microchannels with $5 \mu \mathrm{m}$ constriction. We observed that the transit time for glutaraldehyde treated RBCs (at $20 \mathrm{~ms}$ ) was significantly higher than the transit time for normal cells (at $7 \mathrm{~ms}$ ) (Figure 6). We postulate that, as the deformability of the cell decreases, it takes a longer time for it to pass through the constriction thereby increasing the transit time.
To confirm these results, we used high speed imaging (using Photron FASTCAM at $3000 \mathrm{fps}$ ) to image both the normal RBCs and glutaraldehyde treated RBCs passing through the constriction (Figure 7). The high speed image analysis revealed similar transit times. We recorded an 8 ms transit time for the normal cells and a $19 \mathrm{~ms}$ transit time for the glutaraldehyde treated cells.
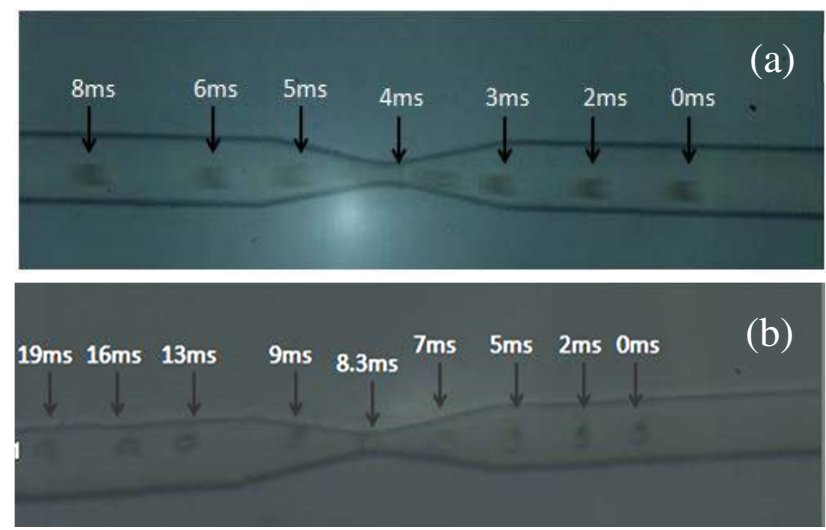

Figure 7: Time lapse images of (a) normal and (b) glutaraldehyde treated RBCs passing through a 200 micron length in constricted channels.

Instantaneous velocity was calculated from the images for the passage of both the cell types through a 200 micron length in the microchannel with constriction. This data suggests that, as the cells move towards the constriction, their velocity increases and the velocity reduces once the cells move out of the constriction. At the constriction, the instantaneous velocity is higher for the normal cells than the glutaraldehyde treated cells, which again confirms that the normal cells are travelling faster as seen in Figure 8 . We believe that the larger increase in the velocity for the normal RBCs is due to their greater deformability as compared to the glutaraldehyde treated cells.

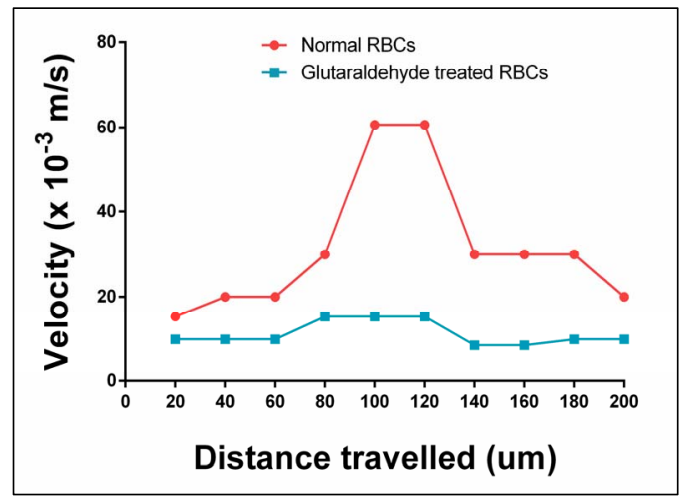

Figure 8: Velocity vs. Distance plot for the passage of the cell through the microchannel

We also observed differences in signal magnitudes between cell populations (normal vs. treated). The normal RBCs had an average signal of $0.18 \mathrm{mV}$ whereas the treated cells had an average signal of $0.23 \mathrm{mV}$. This serves as an excellent indicator of cell properties (see Figure 9). 


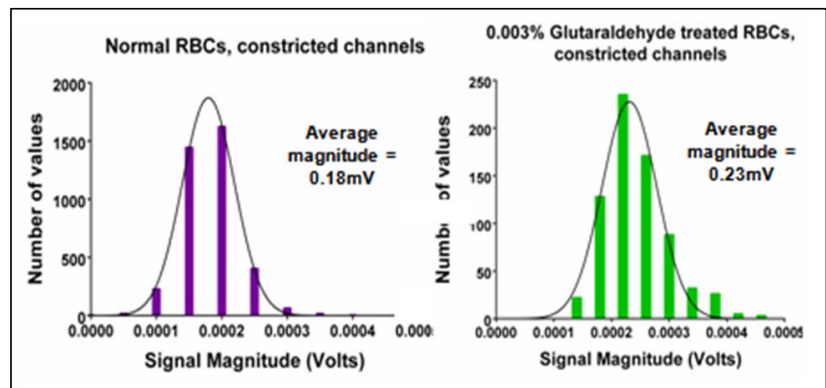

Figure 9: Signal magnitudes of normal RBCs and glutaraldehyde treated RBCs in constricted channels.

As cell porosity is known to increase due to mechanical squeezing, this technique enables exciting future prospects (see Figure 10 for concept) in controlled microinjection of drugs, proteins, DNA, etc. into a cell while probing its biological state electrically, which is cumbersome to achieve in conventional EIS devices.

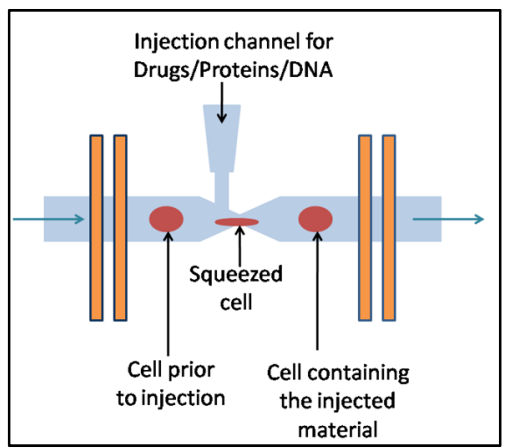

Figure 10: Schematic showing the concept of a controlled microinjection platform to inject drugs/DNA or proteins into cells. The cellular properties can be measured pre and post injection.

\section{CONCLUSIONS}

The novelty of our system lies in the simultaneous and independent measurement of the electrical and mechanical properties of cells. Through EIS signals, the electrical properties of red blood cells were determined. We allow the cells to squeeze through a constriction and correlate the time of transit between two set of electrodes to the mechanical properties of the cells. Using high speed image analysis, we confirm our data. We also demonstrate that our system is capable of distinguishing between treated and untreated cells. In the future, such a system could be used as a cheap, efficient and quick method to diagnose diseases based on electrical and mechanical information from cells.

\section{ACKNOWLEDGEMENTS}

The authors would like to thank the National Nano Fabrication Centre (NNFC) at CeNSE and all its facility technologists for helping us with the device fabrication. The authors also thank the SERB, Department of Science and Technology (DST), Government of India for financially supporting this project.

\section{REFERENCES}

[1] N. Haandbaek, S. C. Burgel, F. Heer, and A. Hierlemann, "Characterization of subcellular morphology of single yeast cells using high frequency microfluidic impedance cytometer," Lab Chip, 14, 369, 2014.

[2] H. Morgan, T. Sun, D. Holmes, S. Gawad, and N. G. Green, "Single-cell dielectric spectroscopy," Journal of Physics D: Applied Physics, 40, pp. 61-70, 2007.

[3] T. Sun and H. Morgan, "Single-cell microfluidic impedance spectroscopy: A review," Microfluidics and Nanofluidics, 8(4), pp. 423-443, 2010.

[4] O. Otto et.al, "Real time deformability cytometry: on-the-fly cell mechanical phenotyping", Nature Methods, 12(3), pp. 199-202, 2015.

[5] D.R. Gossett et. al., "Hydrodynamic stretching of single cells for large population mechanical phenotyping", PNAS, 109(20), pp. 7630-7635, 2012.

[6] J. Chen et.al., "Classification of cell types using a microfluidic device for mechanical and electrical measurement on single cells," Lab Chip, 3174-3181, 2011.

[7] Y. Zheng, E. Shojaei-Baghini, A. Azad, C. Wang and Y. Sun, "High throughput biophysical measurement of human red blood cells," Lab Chip, 12, 2560-2567, 2012.

[8] Y. Zheng, J. Nguyen, C. Wang, and Y. Sun "Electrical measurement of red blood cell deformability on a microfluidic device," Lab Chip, 13, 3275, 2013.

[9] R. S. Ajmani, "Hypertension and hemorheology," Clinical Hemorheology and Microcirculation, 17, 397-420, 1997.

[10] F. C. Mokken, M. Kedaria, C. P. Henny, M. R. Hardeman and A. W. Gelb, "The clinical importance of erythrocyte deformability, a hemorheological parameter," Annals of Hematology, 64, pp. 113-122, 1992.

[11] A. M. Forsyth, J. Wan, W. D. Ristenpart, and H. A. Stone, "The dynamic behavior of chemically "stiffened" red blood cells in microchannel flows," Microvascular Research, 80, 37-43, 2010.

\section{CONTACT}

Karthik Mahesh,

Centre for Nano Science and Engineering (CeNSE),

Indian Institute of Science, Bangalore, India.

Tel: +91-9844760677,

email: karthik@,cense.iisc.ernet.in 